\title{
Profile Distribution of Micronutrient Cations in Soils under Jhum Land in Chandel District of Manipur (India)
}

\author{
Jurist Anal Hrangbung, Herojit Singh Athokpam*, S. Jekendra Singh, \\ K. Nandini Devi and N. Gopimohan Singh
}

College of Agriculture, Central Agricultural University, Imphal - 795004, Manipur, India

*Corresponding author

\section{A B S T R A C T}

\begin{tabular}{|l|}
\hline Ke y w o r d s \\
$\begin{array}{l}\text { DTPA, Micronutrient } \\
\text { cations, Jhum, Soil } \\
\text { profile, Multiple } \\
\text { regressions }\end{array}$ \\
\hline Article Info \\
\hline $\begin{array}{l}\text { Accepted: } \\
\text { 04 October } 2018 \\
\text { Available Online: } \\
\text { 10 November } 2018\end{array}$ \\
\hline \hline
\end{tabular}

Profile distribution of DTPA-extractable micronutrient cations ( $\mathrm{Fe}, \mathrm{Cu}, \mathrm{Mn}$ and $\mathrm{Zn}$ ) and their relationship with various soil physico-chemical properties were studied in twenty jhum land of Chandel district of Manipur. Soils of the upper horizon had higher content of DTPA-extractable micronutrient cations ( $\mathrm{Fe}, \mathrm{Cu}, \mathrm{Mn}$ and $\mathrm{Zn}$ ) than the lower horizons. In the profiles, the value of $\mathrm{Fe}, \mathrm{Cu}, \mathrm{Mn}$ and $\mathrm{Zn}$ varied from 10.27 to $159 \mathrm{mg} \mathrm{kg}^{-1}, 0.04$ to 3.21 $\mathrm{mg} \mathrm{kg}^{-1}, 0.44$ to $38.67 \mathrm{mg} \mathrm{kg}^{-1}$ and 0.10 to $1.67 \mathrm{mg} \mathrm{kg}^{-1}$ soil, respectively. The contents of DTPA- extractable $\mathrm{Fe}$ and $\mathrm{Mn}$ were sufficient in all the profiles, while $\mathrm{Cu}$ was adequate the surface layer $(0-20 \mathrm{~cm})$ in majority of the soil samples with the exception of a few samples. However, 51.25 per cent soils were deficient in DTPA-Zn content in the surface layer. Multiple regression analysis showed that the DTPA-extractable micronutrient cations ( $\mathrm{Fe}, \mathrm{Cu}, \mathrm{Mn}$ and $\mathrm{Zn}$ ) content in the soils were influenced by $\mathrm{pH}, \mathrm{OC}, \mathrm{Mg}, \mathrm{K}, \mathrm{EC}$, CEC and clay to the degree of $54.5,28.8,42.4$ and 46.2 per cent in the surface layer, however, their influenced were significant only by soil $\mathrm{OC}, \mathrm{Mg}$ and $\mathrm{pH}$ in all the profiles.

\section{Introduction}

Micronutrients are the elements required by plants in very small but it is essential for proper growth and development of the plants. $\mathrm{Zn}, \mathrm{Cu}, \mathrm{Mn}, \mathrm{Fe}$ and $\mathrm{B}$ are essential micronutrients for plant growth. Through their involvement in various enzymes and other physiologically active molecules, these micronutrients are important for gene expression, biosynthesis of proteins, nucleic acids, growth substances, chlorophyll and secondary metabolites, metabolism of carbohydrates and lipids, stress tolerance, etc. (Singh, 2004; Rengel, 2007; Gao et al., 2008).
Soil plays a major role in determining the sustainable productivity of an agro-ecosystem. The sustainable productivity of a soil mainly depends upon its ability to supply essential nutrients to the growing plants. The deficiency of micronutrients has become major constraint to productivity, stability and sustainability of soils (Bell and Dell, 2008).

Deficiency of micronutrients may either be primary, due to their low total content or secondary, caused by soil factors reducing their availability to plants. Due to continuous cultivation, soils under particular land use system may affect physico-chemical 
properties which may modify DTPAextractable micronutrient contents and their availability to plants.

Jhum is one of the main forms of agriculture in North Eastern Region (NER) of India. It is also known as shifting cultivation or slash and burn cultivation. Out of the total geographical area of north-eastern region (25.5 million hectares), 2.7 million hectares is under jhum of which $17 \%$ is under use at any given time. Manipur has a geographical area of 22,327 sq. $\mathrm{kms}$ which constitutes $0.7 \%$ of the total land surface of India. Ninety per cent of the total geographical area of the state i.e. 20,097 sq. $\mathrm{kms}$ is covered by hills. In Manipur, jhum is mainly confined in the hill districts. The practice of jhum is not merely done for their sustenance but also it is a traditional method of earning livelihood. Without the adequate availability of micronutrients, it is impossible to get maximum benefit from crop production. The availability of micronutrients to plants is also influenced by the distribution within the soil profile (Singh and Dhankar, 1989). The knowledge of pedogenic distribution of micronutrients is important as plant roots penetrate to the lower layers of the soil and draw a part of the nutrient requirement from the subsurface horizon of the soils. However, there is little or no information regarding status and distribution of micronutrients in soils under jhum in Chandel district of Manipur. Therefore, the present work has been undertaken to assess the distribution of micronutrient cations of the soils under jhum and to find out the relationship between the soil properties and micronutrients.

\section{Materials and Methods}

The district has a geographical area of 496 sq. $\mathrm{km}$ with $2.22 \%$ of the total geographical area of the state. The district $\left(24^{\circ} 40^{\prime} \mathrm{N}\right.$ latitude and $93^{\circ} 50^{\prime}$ E longitude), is located in the southeastern part of Manipur and it experiences hot summer and cold winter. The mean annual temperature exceeds $22^{\circ} \mathrm{C}$ and experiences summer temperature to the range of 35 to $46^{\circ} \mathrm{C}$. The mean annual precipitation varies from 2000 to $2400 \mathrm{~mm}$. The area belongs to warm, humid agro-ecological zone with thermic ecosystem and length of growing period of 300-330 days. The vegetation is predominated by pine including woody and herbaceous species. The soil types of Chandel district are mostly coarser, varying from fine loamy, loamy to sandy in texture and deep in soil depth. Twenty typical soil profiles from different jhum lands of Chandel District, Manipur were exposed and soil samples were collected depth-wise i.e. 0 - 20, 20 - 40, 40 60 and $60-80 \mathrm{~cm}$ in clean polythene bags. Altogether, eighty soil samples were collected. The soil samples were air-dried in the shade, ground and passed through $2 \mathrm{~mm}$ sieve for chemical analysis. The collected samples were processed and analyzed following standard procedures: for mechanical analysis using Bouyoucos hydrometer method (Bouyoucos, 1951); soil $\mathrm{pH}$ and electrical conductivity (EC) using 1:2.5 soil: water suspension (Jackson, 1973), CEC by leaching with 1N $\mathrm{NH}_{4} \mathrm{OAc}$ (Borah et al., 1987) and organic carbon by Walkley and Black's rapid titration method (Walkley and Black, 1934). The soil samples were determined for available nitrogen (Subbiah and Asija, 1956), available P (Bray and Kurtz, 1945), K, Ca and Mg of the soil were extracted with neutral normal ammonium acetate as described by Gupta (2006) and DTPA-extractable $\mathrm{Zn}, \mathrm{Cu}, \mathrm{Mn}$ and Fe following standard procedures as outlined by (Lindsay and Norvell, 1978). The relationship between various soil properties and micro-nutrients distribution were established by using simple correlation coefficient. Multiple regression equations were computed between DTPA-extractable micronutrients and soil properties by adopting statistical procedures (Panse and Sukhatme, 1961). 


\section{Results and Discussion}

The physico-chemical properties as well as DTPA- extractable $\mathrm{Fe}, \mathrm{Cu}, \mathrm{Mn}$ and $\mathrm{Zn}$ are presented in Table 1 and 2. There was no definite pattern in the distribution of sand, silt and clay soil profiles. Sand, silt and clay fractions in the soils ranged from 19.5 to 82.5 , 2.5 to 48.8 and 10.5 to 62.3 per cent, respectively. The samples were very strongly acidic ( $\mathrm{pH} 4.67)$ to slightly acidic ( $\mathrm{pH}$ 6.29) in reaction. The EC values of the soils ranged from 0.01 to $0.35 \mathrm{dSm}^{-1}$. OC content of the studied soil samples ranged from 0.9 to $23.1 \mathrm{~g}$ $\mathrm{kg}^{-1}$. CEC varied from 4.6 to $16.8\left[\mathrm{cmol}\left(\mathrm{p}^{+}\right)\right.$ $\mathrm{kg}^{-1}$ ] soil.

The $\mathrm{N}, \mathrm{P}_{2} \mathrm{O}_{5}$ and $\mathrm{K}_{2} \mathrm{O}$ content in the soil samples varied from 60.63 to $551.94 \mathrm{~kg} \mathrm{ha}^{-1}$, 7.75 to $34.05 \mathrm{~kg} \mathrm{ha}^{-1}$ and 40.32 to $365.10 \mathrm{~kg}$ $\mathrm{ha}^{-1}$, respectively. Exchangeable $\mathrm{Ca}^{++}$content varied from 0.1 to $5.6\left[\mathrm{cmol}(\mathrm{p}+) \mathrm{kg}^{-1}\right]$ and $\mathrm{Mg}^{++}$from 0.1 to $5.6\left[\mathrm{cmol}(\mathrm{p}+) \mathrm{kg}^{-1}\right]$. Low content of these bases in the soils might be due to the leaching losses as the area is high in rainfall. The content of these nutrients decreased with increase in depth in most of the studied profiles. Similar results were also reported by Athokpam et al., (2016) and Athokpam et al., (2018) in the soils of Ukhrul and Tamenglong districts of Manipur, respectively.

\section{Iron $(\mathbf{F e})$}

DTPA-extractable $\mathrm{Fe}$ in the soil profiles varied from 10.27 to $159.00 \mathrm{mg} \mathrm{kg}^{-1}$ soil in the jhum soils of Chandel district of Manipur. The high amount of $\mathrm{Fe}$ content in the soil profiles might be the leaching loss of exchangeable bases from the surface soils, thus increasing the acidity, which in turn increasing the $\mathrm{Fe}$ content in the soils. Considering $4.5 \mathrm{mg} \mathrm{kg}^{-1}$ as critical limit as suggested by Lindsay and Norvell (1978), all the soils had sufficient amount of Fe. It showed significant regression coefficient with $\mathrm{OC}\left(8.968^{*}\right)$ and $\mathrm{Cu}$ $\left(28.598^{*}\right)$ in the $2^{\text {nd }}$ layer and $\mathrm{Mg}\left(11.638^{*}\right)$ and $\mathrm{Cu}\left(48.849^{*}\right)$ in the $3^{\text {rd }}$ layer. Multiple correlation and regression analyses indicated that 54.5, 55.5, 55.9 and 32.2 per cent variability in the DTPA-extractable Fe in the profiles was due to the simultaneous effect of $\mathrm{pH}, \mathrm{OC}, \mathrm{Cu}, \mathrm{Mn}, \mathrm{Mg}$ and $\mathrm{Zn}$ in the soils. The surface layer soil contained more available Fe than those in lower depth and showed a decreasing pattern along with depth except for few samples. These findings are in agreement with earlier works of Sen et al., (1997), Gupta et al., (2003), Sharma et al., (2003), Pati and Mukhopadhyay (2011), Athokpam et al., (2013), Athokpam et al., (2016) and Athokpam et al., (2018).

\section{Copper (Cu)}

DTPA-extractable $\mathrm{Cu}$ content in the profiles ranged from 0.04 to $3.21 \mathrm{mg} \mathrm{kg}^{-1}$ soils. Considering $0.20 \mathrm{mg} \mathrm{Cu} \mathrm{kg}^{-1}$ soil as critical level (Lindsay and Norvell, 1978), all the samples except a few in the sub-surface horizons, were well supplied with available $\mathrm{Cu}$. Similar findings were also made by Sen et al., (1997). DTPA-extractable $\mathrm{Cu}$ content was higher in the surface soils and decreased gradually in most of the profiles. This might be due to higher biological activity and organic carbon content in the surface layer (Murthy et al., 1997). Similar results were also reported by various researchers (Gupta et al., 2003, Verma et al., 2007a, Verma et al., 2007b, Athokpam et al., (2013), Athokpam et al., (2016) and Athokpam et al., (2018)). However, irregular distribution of DTPAextractable $\mathrm{Cu}$ with depth was observed in a few samples. Similar findings were made by Sangwan et al., (1993), Kumar et al., (1996) and Satyavathi and Reddy (2004). It showed significant multiple regression coefficient with $\mathrm{Fe}\left(0.008^{*}\right)$ in the second layer, $\mathrm{Fe}\left(0.005^{*}\right)$ and $\mathrm{Zn}\left(0.663^{*}\right)$ in the third layer and $\mathrm{Zn}$ $\left(0.986^{*}\right)$ in the fourth layer. 
Table.1 Physico-chemical properties of the soil profiles

\begin{tabular}{|c|c|c|c|c|c|c|c|c|c|c|c|c|c|}
\hline $\begin{array}{l}\text { Depth } \\
\text { (cm) }\end{array}$ & $\begin{array}{l}\text { Clay } \\
(\%)\end{array}$ & $\begin{array}{l}\text { Silt } \\
(\%)\end{array}$ & $\begin{array}{l}\text { Sand } \\
(\%)\end{array}$ & Texture & $\mathbf{P h}$ & $\begin{array}{l}\mathrm{EC} \\
\left(\mathrm{dSm}^{-1}\right)\end{array}$ & $\begin{array}{l}\text { CEC [cmol } \\
\left.\left(p^{+}\right) \mathrm{kg}^{-1}\right]\end{array}$ & $\begin{array}{l}\mathbf{O C} \\
(\mathrm{g} \\
\left.\mathrm{kg}^{-1}\right)\end{array}$ & $\begin{array}{l}\mathbf{C a} \\
{[\mathrm{cmol}} \\
\left.\left(\mathbf{p}^{+}\right) \mathbf{k g}^{-1}\right]\end{array}$ & \begin{tabular}{|l}
$\mathrm{Mg}$ \\
{$[\mathrm{cmol}$} \\
$\left(\mathrm{p}^{+}\right)$ \\
$\left.\mathrm{kg}^{-1}\right]$ \\
\end{tabular} & $\begin{array}{l}\mathbf{N} \\
(\mathbf{k g} \\
\left.\mathrm{ha}^{-1}\right)\end{array}$ & $\begin{array}{l}\mathrm{P}_{2} \mathrm{O}_{5} \\
(\mathbf{k g} \\
\left.\mathrm{ha}^{-1}\right)\end{array}$ & $\begin{array}{l}\mathrm{K}_{2} \mathbf{O} \\
(\mathbf{k g} \\
\left.\mathbf{h a}^{-1}\right)\end{array}$ \\
\hline \multicolumn{14}{|c|}{ 1.Panchai } \\
\hline $0-20$ & 27.5 & 17.5 & 55 & SCL & 5.3 & 0.18 & 6.4 & 7.8 & 0.5 & 0.4 & 100.35 & 20.04 & 218.21 \\
\hline $20-40$ & 45.7 & 12.5 & 41.8 & $\mathrm{C}$ & 5.40 & 0.05 & 6 & 5.1 & 1.0 & 0.4 & 314.66 & 15.31 & 153.48 \\
\hline $40-60$ & 43 & 15 & 42 & $\mathrm{C}$ & 5.51 & 0.05 & 5.8 & 4.2 & 0.2 & 0.3 & 225.79 & 10.99 & 112.63 \\
\hline $60-80$ & 36.4 & 25 & 38.6 & $\mathrm{CL}$ & 5.49 & 0.03 & 5.6 & 4.5 & 0.1 & 0.1 & 100.35 & 9.55 & 91.12 \\
\hline \multicolumn{14}{|c|}{ 2.Charangching Khunkha } \\
\hline $0-20$ & 47.3 & 5.0 & 47.7 & $\mathrm{SC}$ & 5.34 & 0.14 & 10.4 & 9.3 & 0.5 & 3.4 & 200.70 & 15.11 & 365.1 \\
\hline $20-40$ & 58.2 & 17.5 & 24.3 & $\mathrm{C}$ & 5.45 & 0.03 & 11.4 & 3.0 & 1.5 & 0.7 & 188.16 & 10.63 & 266.38 \\
\hline $40-60$ & 60.9 & 12.5 & 26.6 & $\mathrm{C}$ & 5.50 & 0.04 & 9.4 & 3.7 & 0.7 & 0.5 & 188.16 & 13.51 & 192.86 \\
\hline $60-80$ & 56.6 & 12.5 & 30.9 & $\mathrm{C}$ & 5.69 & 0.03 & 9.2 & 2.3 & 2.8 & 0.4 & 175.61 & 9.91 & 155.37 \\
\hline \multicolumn{14}{|c|}{ 3.Charangching Khullen } \\
\hline $0-20$ & 36.2 & 15 & 48.8 & $\mathrm{SC}$ & 5.63 & 0.15 & 11.4 & 23.1 & 2.9 & 2.7 & 413.95 & 20.72 & 305.62 \\
\hline $20-40$ & 37.7 & 17.5 & 44.8 & $\mathrm{CL}$ & 5.61 & 0.06 & 11.2 & 10.8 & 5.6 & 0.5 & 238.34 & 16.75 & 166.25 \\
\hline $40-60$ & 37 & 10 & 53 & $\mathrm{SC}$ & 5.99 & 0.05 & 10.8 & 8.7 & 4.5 & 3.5 & 175.63 & 13.87 & 136.68 \\
\hline $60-80$ & 34.6 & 17.5 & 47.9 & SCL & 6.15 & 0.03 & 10.8 & 3.0 & 4.5 & 2.3 & 163.07 & 7.75 & 104.29 \\
\hline \multicolumn{14}{|c|}{ 4. Hnatham } \\
\hline $0-20$ & 33.2 & 22.5 & 44.3 & $\mathrm{CL}$ & 5.43 & 0.05 & 9.4 & 15.4 & 3.8 & 3.1 & 263.42 & 17.11 & 163.97 \\
\hline $20-40$ & 36.6 & 25 & 38.4 & $\mathrm{CL}$ & 5.52 & 0.03 & 9.2 & 10.2 & 2.5 & 3.0 & 250.88 & 14.23 & 148.51 \\
\hline $40-60$ & 30.7 & 32.5 & 36.8 & $\mathrm{CL}$ & 5.91 & 0.02 & 5.2 & 6.5 & 2.7 & 2.5 & 213.24 & 13.15 & 139.91 \\
\hline $60-80$ & 42.5 & 15 & 42.5 & $\mathrm{C}$ & 5.97 & 0.03 & 5 & 6.0 & 2.2 & 2.1 & 163.07 & 10.27 & 161.28 \\
\hline \multicolumn{14}{|c|}{ 5.Hnahringkhu } \\
\hline $0-20$ & 11.6 & 10 & 78.4 & SL & 5.5 & 0.08 & 9.4 & 6.9 & 4.5 & 5.6 & 551.94 & 16.39 & 198.51 \\
\hline $20-40$ & 25 & 10 & 65 & SCL & 5.67 & 0.16 & 8.4 & 8.1 & 4.0 & 3.9 & 263.42 & 13.15 & 202.68 \\
\hline $40-60$ & 27.5 & 2.5 & 70 & SCL & 5.88 & 0.25 & 7.4 & 6.9 & 4.1 & 3.7 & 137.98 & 11.71 & 125.40 \\
\hline $60-80$ & 25.9 & 15 & 59.1 & SCL & 6.14 & 0.07 & 7 & 4.5 & 3.9 & 3.2 & 112.90 & 9.55 & 109.80 \\
\hline \multicolumn{14}{|c|}{ 6.Lambung } \\
\hline $0-20$ & 27.5 & 17.5 & 55 & SCL & 5.73 & 0.05 & 12.4 & 5.7 & 5.5 & 3.0 & 314.66 & 15.31 & 181.04 \\
\hline $20-40$ & 25 & 5 & 70 & SCL & 6.08 & 0.26 & 13.2 & 6.3 & 5.5 & 3.8 & 163.07 & 13.87 & 196.63 \\
\hline $40-60$ & 10.5 & 7 & 82.5 & LS & 6.1 & 0.02 & 12.8 & 2.1 & 5.4 & 4.9 & 150.53 & 11.35 & 160.74 \\
\hline $60-80$ & 16.6 & 15 & 68.4 & SL & 6.29 & 0.02 & 13 & 1.5 & 5.1 & 3.0 & 75.26 & 10.27 & 128.62 \\
\hline \multicolumn{14}{|c|}{ 7.Mantripantha } \\
\hline $0-20$ & 30 & 20 & 50 & SCL & 5.68 & 0.1 & 7.8 & 18.3 & 2.5 & 1.5 & 275.97 & 23.96 & 355.07 \\
\hline $20-40$ & 42.7 & 15 & 42.3 & $\mathrm{C}$ & 5.72 & 0.06 & 7.2 & 9.6 & 2.7 & 1.0 & 275.97 & 18.92 & 240.10 \\
\hline $40-60$ & 41.7 & 15 & 43.3 & $\mathrm{C}$ & 5.70 & 0.04 & 7.6 & 4.8 & 2.4 & 1.9 & 263.42 & 10.63 & 210 \\
\hline $60-80$ & 43 & 15 & 42 & C & 5.79 & 0.04 & 6.8 & 3.9 & 2.0 & 2.1 & 213.25 & 9.91 & 190.57 \\
\hline $\begin{array}{l}\text { Depth } \\
\text { (cm) }\end{array}$ & $\begin{array}{l}\text { Clay } \\
(\%)\end{array}$ & $\begin{array}{l}\text { Silt } \\
(\%)\end{array}$ & $\begin{array}{l}\text { Sand } \\
(\%)\end{array}$ & Texture & $\mathrm{pH}$ & $\begin{array}{l}\mathrm{EC} \\
(\mathrm{dSm} \\
\left.{ }^{1}\right)\end{array}$ & $\begin{array}{l}\text { CEC } \\
{\left[\mathrm{cmol}^{+}\left(\mathrm{p}^{+}\right)\right.} \\
\left.\mathrm{kg}^{-1}\right]\end{array}$ & $\begin{array}{l}\mathrm{OC} \\
(\mathrm{g} \\
\left.\mathrm{kg}^{-1}\right)\end{array}$ & $\begin{array}{l}\mathrm{Ca} \\
{[\mathrm{cmol}} \\
\left.\left(\mathrm{p}^{+}\right) \mathrm{kg}^{-1}\right]\end{array}$ & $\begin{array}{l}\mathrm{Mg} \\
{\left[\mathrm{cmol}^{+}\left(\mathrm{p}^{+}\right)\right.} \\
\left.\mathrm{kg}^{-1}\right]\end{array}$ & $\begin{array}{l}\mathrm{N} \\
(\mathrm{kg} \\
\left.\mathrm{ha}^{-1}\right)\end{array}$ & $\begin{array}{l}\mathrm{P}_{2} \mathrm{O}_{5} \\
\left(\mathrm{kgha}^{-1}\right)\end{array}$ & $\begin{array}{l}\mathrm{K}_{2} \mathrm{O} \\
\left(\mathrm{kgha}^{-1}\right)\end{array}$ \\
\hline \multicolumn{14}{|c|}{ 8.Modi } \\
\hline $0-20$ & 37 & 15.5 & 47.5 & SC & 5.69 & 0.06 & 10.6 & 15.2 & 3.9 & 1.2 & 238.34 & 23.60 & 273.26 \\
\hline $20-40$ & 40 & 9.75 & 50.25 & SC & 5.82 & 0.03 & 9.8 & 8.7 & 4.2 & 0.9 & 225.79 & 20.72 & 143.40 \\
\hline $40-60$ & 44.1 & 10 & 45.9 & SC & 6.01 & 0.02 & 9.8 & 1.8 & 4.5 & 0.8 & 150.53 & 18.92 & 110.53 \\
\hline $60-80$ & 29.8 & 12.5 & 57.7 & SCL & 6.19 & 0.02 & 8.2 & 0.9 & 4.2 & 0.7 & 137.98 & 17.11 & 108.08 \\
\hline \multicolumn{14}{|c|}{ 9.Angthi } \\
\hline $0-20$ & 37 & 20 & 43 & CL & 5.43 & 0.06 & 8.8 & 8.7 & 2.4 & 2.0 & 175.62 & 29 & 310.46 \\
\hline $20-40$ & 41.5 & 35 & 23.5 & C & 5.35 & 0.03 & 7.2 & 8.4 & 2.1 & 1.9 & 200.70 & 21.08 & 194.47 \\
\hline $40-60$ & 38.2 & 20 & 41.8 & CL & 5.6 & 0.01 & 9 & 6.3 & 2.0 & 1.9 & 163.07 & 16.39 & 177.40 \\
\hline $60-80$ & 45.7 & 12.5 & 41.8 & $\mathrm{C}$ & 5.9 & 0.02 & 8 & 3.1 & 1.9 & 0.9 & 150.53 & 17.47 & 138.83 \\
\hline \multirow{2}{*}{\multicolumn{14}{|c|}{$\begin{array}{l}\text { 10.Lamphou Charu } \\
0.06 \quad 6.8\end{array}$}} \\
\hline & & & & & 5.61 & & 6.4 & 13.8 & 5.3 & 4.4 & 275.97 & 25.04 & 270.61 \\
\hline
\end{tabular}




\begin{tabular}{|c|c|c|c|c|c|c|c|c|c|c|c|c|c|}
\hline $20-40$ & 29.1 & 40 & 30.9 & CL & 5.67 & 0.12 & 4.6 & 9.5 & 4.8 & 3.9 & 225.79 & 18.92 & 250.52 \\
\hline $40-60$ & 24.1 & 25 & 50.9 & SCL & 5.79 & 0.05 & 5.2 & 4.5 & 4.2 & 3.3 & 137.98 & 16.75 & 152.28 \\
\hline $60-80$ & 36.6 & 25 & 38.4 & $\mathrm{CL}$ & 6.12 & 0.04 & 5.2 & 3.5 & 4.0 & 3.1 & 87.81 & 15.67 & 150.93 \\
\hline \multicolumn{14}{|c|}{ 11.Lamphou Pasna } \\
\hline $0-20$ & 28 & 32.5 & 39.5 & $\mathrm{CL}$ & 5.22 & 0.04 & 6.2 & 7.2 & 0.5 & 0.2 & 200.70 & 18.92 & 225.92 \\
\hline $20-40$ & 30.5 & 40 & 29.5 & $\mathrm{CL}$ & 5.44 & 0.03 & 6.0 & 6.9 & 0.8 & 0.7 & 188.16 & 14.59 & 276.86 \\
\hline $40-60$ & 21.4 & 32.5 & 46.1 & $\mathrm{~L}$ & 5.49 & 0.03 & 5.6 & 4.8 & 0.4 & 1.1 & 175.62 & 10.63 & 98.92 \\
\hline $60-80$ & 26.4 & 35 & 38.6 & $\mathrm{~L}$ & 5.63 & 0.03 & 5.4 & 1.7 & 0.7 & 1.7 & 175.62 & 9.91 & 137.49 \\
\hline \multicolumn{14}{|c|}{ 12.Liwa Sarei } \\
\hline $0-20$ & 33 & 35 & 32 & CL & 4.67 & 0.16 & 7.0 & 9.9 & 0.9 & 0.1 & 175.62 & 34.05 & 120.28 \\
\hline $20-40$ & 50.5 & 25 & 24.5 & $\mathrm{C}$ & 4.97 & 0.12 & 6.4 & 5.7 & 0.2 & 1.9 & 163.07 & 24.68 & 235.06 \\
\hline $40-60$ & 53 & 25 & 22 & $\mathrm{C}$ & 5.42 & 0.03 & 6 & 2.4 & 1.0 & 0.7 & 137.98 & 17.47 & 216.79 \\
\hline $60-80$ & 53 & 27.5 & 19.5 & $\mathrm{C}$ & 5.41 & 0.02 & 5.8 & 1.8 & 0.6 & 0.8 & 100.35 & 12.79 & 197.30 \\
\hline \multicolumn{14}{|c|}{ 13.Thotchanram } \\
\hline $0-20$ & 28.2 & 17.5 & 54.3 & SCL & 5.21 & 0.05 & 6.4 & 5.9 & 2.2 & 2.9 & 150.53 & 28.28 & 152.54 \\
\hline $20-40$ & 45.7 & 12.5 & 41.8 & $\mathrm{C}$ & 5.43 & 0.05 & 6.0 & 4.8 & 1.3 & 1.1 & 137.98 & 18.92 & 154.28 \\
\hline $40-60$ & 23.2 & 30 & 46.8 & $\mathrm{~L}$ & 5.3 & 0.04 & 5.8 & 3.9 & 1.1 & 1.7 & 100.35 & 11.35 & 127.40 \\
\hline $60-80$ & 41.7 & 15 & 43.3 & $\mathrm{C}$ & 5.51 & 0.03 & 5.6 & 1.1 & 1.0 & 3.5 & 87.81 & 10.99 & 111.39 \\
\hline \multicolumn{14}{|c|}{ 14.Cheengkhu } \\
\hline $0-20$ & 29.3 & 17.5 & 53.2 & SCL & 5.07 & 0.06 & 8.6 & 7.2 & 0.4 & 2.1 & 539.39 & 21.08 & 278.75 \\
\hline $20-40$ & 36 & 17.5 & 46.5 & $\mathrm{SC}$ & 5.19 & 0.04 & 8.2 & 6.3 & 0.5 & 1.7 & 238.34 & 14.59 & 167.81 \\
\hline 40-60 & 29.8 & 17.5 & 52.7 & SCL & 5.24 & 0.04 & 7.8 & 3.8 & 0.2 & 1.3 & 175.62 & 10.27 & 147.83 \\
\hline $60-80$ & 27.3 & 17.5 & 55.2 & SCL & 5.53 & 0.03 & 7.6 & 2.2 & 0.1 & 1.2 & 100.35 & 9.19 & 132.24 \\
\hline \multicolumn{14}{|c|}{ 15.Angkhel Chayang } \\
\hline $0-20$ & 43 & 24.1 & 32.9 & $\mathrm{C}$ & 4.88 & 0.05 & 7.2 & 9.0 & 1.2 & 1.7 & 514.30 & 26.48 & 263.64 \\
\hline $20-40$ & 42 & 33.8 & 24.2 & $\mathrm{C}$ & 5.1 & 0.03 & 9.4 & 8.1 & 1.2 & 0.3 & 363.78 & 22.88 & 221.22 \\
\hline $40-60$ & 48 & 24.1 & 27.9 & $\mathrm{C}$ & 5.3 & 0.04 & 7.4 & 4.8 & 0.8 & 0.2 & 301.06 & 16.03 & 194.07 \\
\hline $60-80$ & 33.5 & 48.8 & 17.7 & SiCL & 5.34 & 0.03 & 6.8 & 1.2 & 0.5 & 0.2 & 275.96 & 12.79 & 146.23 \\
\hline \multicolumn{14}{|c|}{ 16.Japhou } \\
\hline $0-20$ & 46.6 & 15 & 38.4 & $\mathrm{C}$ & 5.23 & 0.08 & 9.6 & 12 & 0.7 & 0.2 & 464.13 & 27.20 & 40.32 \\
\hline $20-40$ & 41.6 & 15 & 43.4 & C & 5.26 & 0.05 & 9.4 & 8.4 & 0.8 & 0.3 & 288.51 & 20.36 & 43.95 \\
\hline $40-60$ & 44.1 & 15 & 40.9 & $\mathrm{C}$ & 5.49 & 0.04 & 7.4 & 6.9 & 0.6 & 0.3 & 288.51 & 16.39 & 71.90 \\
\hline $60-80$ & 44.1 & 12.5 & 43.4 & $\mathrm{C}$ & 5.81 & 0.02 & 7.2 & 3.5 & 0.4 & 0.5 & 313.60 & 17.47 & 53.63 \\
\hline \multicolumn{14}{|c|}{ 17.Monsang Pantha } \\
\hline $0-20$ & 59.8 & 5 & 35.2 & $\mathrm{C}$ & 5.16 & 0.06 & 11.6 & 12.7 & 0.7 & 1.3 & 326.14 & 29.72 & 225.24 \\
\hline $20-40$ & 44.8 & 17.5 & 37.7 & $\mathrm{C}$ & 5.18 & 0.06 & 11.4 & 9.6 & 0.6 & 1.4 & 275.96 & 20.36 & 273.5 \\
\hline $40-60$ & 62.3 & 5 & 32.7 & $\mathrm{C}$ & 5.23 & 0.04 & 11 & 6.2 & 0.6 & 0.4 & 275.96 & 16.75 & 228.21 \\
\hline $60-80$ & 47.3 & 12.5 & 40.2 & $\mathrm{C}$ & 5.55 & 0.03 & 10.8 & 3.3 & 2.5 & 2.3 & 60.63 & 10.63 & 180.23 \\
\hline \multicolumn{14}{|c|}{ 18.Thangkin } \\
\hline $0-20$ & 42.8 & 17.5 & 39.7 & $\mathrm{C}$ & 4.96 & 0.19 & 13.6 & 13.5 & 0.8 & 0.4 & 376.32 & 32.25 & 276.85 \\
\hline $20-40$ & 42.8 & 17.5 & 39.7 & $\mathrm{C}$ & 5.24 & 0.09 & 13.2 & 7.4 & 1.1 & 0.2 & 351.23 & 27.20 & 233.72 \\
\hline $40-60$ & 46.9 & 22.5 & 30.6 & $\mathrm{C}$ & 5.25 & 0.06 & 12.8 & 3.4 & 0.3 & 0.1 & 288.51 & 13.87 & 219.88 \\
\hline $60-80$ & 49.4 & 22.5 & 28.1 & $\mathrm{C}$ & 5.34 & 0.03 & 11.4 & 3.6 & 0.2 & 0.1 & 188.16 & 10.27 & 156.04 \\
\hline \multicolumn{14}{|c|}{ 19.Chumthar } \\
\hline $0-20$ & 42.5 & 20 & 37.5 & $\mathrm{C}$ & 4.67 & 0.06 & 12.6 & 7.9 & 1.3 & 1.5 & 200.70 & 26.84 & 315.30 \\
\hline $20-40$ & 39.1 & 20 & 40.9 & CL & 5 & 0.05 & 11.8 & 8.4 & 1.2 & 1.0 & 188.16 & 21.44 & 235.33 \\
\hline $40-60$ & 41 & 22.5 & 36.5 & C & 5.11 & 0.04 & 11.4 & 3.7 & 1.1 & 0.8 & 175.62 & 18.92 & 208.05 \\
\hline $60-80$ & 36.6 & 20.9 & 42.5 & CL & 5.29 & 0.03 & 10.0 & 3 & 1.0 & 0.6 & 175.62 & 15.67 & 149.05 \\
\hline \multicolumn{14}{|c|}{ 20.Khongion } \\
\hline $0-20$ & 40.7 & 20 & 39.3 & $\mathrm{C}$ & 5.1 & 0.35 & 16.8 & 21.3 & 2.4 & 0.4 & 162.72 & 27.56 & 304.13 \\
\hline $20-40$ & 48.2 & 12.5 & 39.3 & C & 5.08 & 0.15 & 16.2 & 8.1 & 2 & 2.8 & 213.25 & 22.52 & 159.94 \\
\hline $40-60$ & 48.2 & 10 & 41.8 & $\mathrm{C}$ & 5.14 & 0.03 & 15.8 & 6.6 & 0.4 & 1.2 & 188.16 & 16.39 & 126.74 \\
\hline $60-80$ & 53.2 & 2.5 & 44.3 & $\mathrm{C}$ & 5.21 & 0.03 & 16.4 & 3.9 & 0.4 & 1.5 & 137.98 & 13.87 & 111.96 \\
\hline
\end{tabular}


Table.2 Distribution of available DTPA - Extractable micronutrients of soils

\begin{tabular}{|c|c|c|c|c|c|c|c|c|c|}
\hline Depth (cm) & $\mathrm{Fe}$ & $\mathbf{C u}$ & Mn & $\mathrm{Zn}$ & Depth (cm) & Fe & $\mathrm{Cu}$ & Mn & $\mathrm{Zn}$ \\
\hline \multicolumn{5}{|l|}{ 1.Panchai } & \multicolumn{5}{|c|}{ 2.Charangching Khunkha } \\
\hline $0-20$ & 51.8 & 0.77 & 11.48 & 0.65 & $0-20$ & 36.19 & 0.85 & 14.91 & 0.92 \\
\hline $20-40$ & 19.73 & 0.43 & 4.97 & 0.29 & $20-40$ & 13.21 & 0.78 & 13.64 & 0.56 \\
\hline $40-60$ & 14.96 & 0.22 & 2.73 & 0.14 & $40-60$ & 10.27 & 0.32 & 7.21 & 0.53 \\
\hline $60-80$ & 17.01 & 0.19 & 6.22 & 0.10 & $60-80$ & 11.50 & 0.14 & 5.54 & 0.3 \\
\hline \multicolumn{5}{|c|}{ 3.Charangching Khullen } & \multicolumn{5}{|l|}{ 4.Hnatham } \\
\hline $0-20$ & 122.08 & 0.35 & 16.54 & 1.12 & $0-20$ & 130.21 & 1.48 & 30.53 & 0.91 \\
\hline $20-40$ & 90.74 & 0.25 & 19.39 & 0.49 & $20-40$ & 105.24 & 0.84 & 29.50 & 0.76 \\
\hline $40-60$ & 65.84 & 0.18 & 19.84 & 0.38 & $40-60$ & 68.78 & 0.65 & 23.35 & 0.54 \\
\hline $60-80$ & 41.13 & 0.04 & 10.40 & 0.17 & $60-80$ & 49.07 & 0.20 & 19.93 & 0.48 \\
\hline \multicolumn{5}{|c|}{ 5.Hnahringkhu } & \multicolumn{5}{|l|}{ 6.Lambung } \\
\hline $0-20$ & 85.69 & 1.05 & 15.63 & 0.65 & $0-20$ & 112.92 & 1.21 & 26.25 & 0.96 \\
\hline $20-40$ & 82.26 & 0.75 & 19.71 & 0.56 & $20-40$ & 88.69 & 0.47 & 16.80 & 0.75 \\
\hline $40-60$ & 80.69 & 0.52 & 18.87 & 0.58 & $40-60$ & 89.96 & 0.22 & 15.28 & 0.43 \\
\hline $60-80$ & 69.77 & 0.51 & 15.37 & 0.46 & $60-80$ & 50.92 & 0.45 & 13.06 & 0.39 \\
\hline \multicolumn{5}{|c|}{ 7.Mantripantha } & \multicolumn{5}{|l|}{ 8.Modi } \\
\hline $0-20$ & 159 & 3.21 & 14.52 & 1.05 & $0-20$ & 110.16 & 1.58 & 37.31 & 0.86 \\
\hline $20-40$ & 156.98 & 2.54 & 14.10 & 0.97 & $20-40$ & 59.66 & 0.57 & 14.23 & 0.79 \\
\hline $40-60$ & 154.48 & 1.87 & 9.33 & 0.99 & $40-60$ & 34.26 & 0.50 & 11.91 & 0.68 \\
\hline $60-80$ & 105.54 & 1.70 & 11.5 & 0.75 & $60-80$ & 33.36 & 0.44 & 9.74 & 0.49 \\
\hline \multicolumn{5}{|l|}{ 9.Angthi } & \multicolumn{5}{|c|}{ 10.Lamphou Charu } \\
\hline $0-20$ & 52.92 & 0.19 & 27.24 & 0.51 & $0-20$ & 65.24 & 1.84 & 31.36 & 0.78 \\
\hline $20-40$ & 50.59 & 0.54 & 25.32 & 1.22 & $20-40$ & 46.96 & 0.65 & 24.91 & 1.59 \\
\hline $40-60$ & 50.20 & 0.47 & 24.81 & 0.57 & $40-60$ & 30.01 & 0.97 & 20.41 & 1.06 \\
\hline $60-80$ & 48.74 & 0.46 & 13.05 & 0.40 & $60-80$ & 17.83 & 1.03 & 20.06 & 0.64 \\
\hline \multicolumn{5}{|c|}{ 11.Lamphou Pasna } & \multicolumn{5}{|l|}{ 12.Liwa Sarei } \\
\hline $0-20$ & 77.01 & 2.06 & 6.21 & 0.47 & $0-20$ & 53.19 & 0.85 & 10.53 & 1.12 \\
\hline $20-40$ & 25.84 & 1.15 & 15.89 & 0.30 & $20-40$ & 52.62 & 0.33 & 2.78 & 0.83 \\
\hline $40-60$ & 19.28 & 0.76 & 10.30 & 0.30 & $40-60$ & 51.19 & 0.65 & 1.52 & 0.97 \\
\hline $60-80$ & 19.10 & 0.23 & 9.19 & 0.13 & $60-80$ & 49.62 & 0.35 & 0.86 & 0.65 \\
\hline \multicolumn{5}{|c|}{ 13. Thotchanram } & \multicolumn{5}{|l|}{ 14. Cheengkhu } \\
\hline $0-20$ & 36.70 & 1.63 & 3.70 & 0.87 & $0-20$ & 87.18 & 1.19 & 27.25 & 1.43 \\
\hline $20-40$ & 46.55 & 0.32 & 2.37 & 0.49 & $20-40$ & 44.61 & 0.98 & 7.56 & 0.45 \\
\hline $40-60$ & 43.57 & 0.55 & 4.39 & 0.53 & $40-60$ & 43.38 & 0.58 & 14.14 & 0.39 \\
\hline $60-80$ & 34.32 & 0.19 & 3.14 & 0.48 & $60-80$ & 36.55 & 0.51 & 25.90 & 0.11 \\
\hline \multicolumn{5}{|c|}{ 15. Angkhel Chayang } & \multicolumn{5}{|l|}{ 16. Japhou } \\
\hline $0-20$ & 49.71 & 0.33 & 11.53 & 1.06 & $0-20$ & 63.16 & 1.23 & 13.57 & 0.61 \\
\hline $20-40$ & 26.85 & 0.76 & 11.43 & 0.96 & $20-40$ & 57.68 & 0.36 & 12.51 & 0.47 \\
\hline $40-60$ & 21.89 & 0.60 & 11.39 & 0.73 & $40-60$ & 56.76 & 0.32 & 38.67 & 0.45 \\
\hline $60-80$ & 23.42 & 0.78 & 8.48 & 0.53 & $60-80$ & 53.24 & 0.28 & 25.12 & 0.49 \\
\hline \multicolumn{5}{|c|}{ 17. Monsang Pantha } & \multicolumn{5}{|l|}{ 18. Thangkin } \\
\hline $0-20$ & 113.01 & 0.61 & 21.99 & 1.67 & $0-20$ & 44.13 & 0.80 & 9.31 & 1.48 \\
\hline $20-40$ & 83.87 & 1.04 & 26.12 & 1.38 & $20-40$ & 37.15 & 0.72 & 5.67 & 0.83 \\
\hline $40-60$ & 52.92 & 0.77 & 21.52 & 1.23 & $40-60$ & 36.98 & 0.60 & 5.66 & 0.51 \\
\hline $60-80$ & 33.88 & 0.54 & 23.71 & 0.51 & $60-80$ & 34.13 & 0.10 & 4.20 & 0.33 \\
\hline \multicolumn{5}{|l|}{ 19. Chumthar } & 20. Khongjon & & & & \\
\hline $0-20$ & 60.91 & 0.37 & 1.37 & 0.97 & $0-20$ & 80.21 & 0.85 & 15.63 & 1.66 \\
\hline $20-40$ & 48.47 & 0.06 & 0.44 & 0.56 & $20-40$ & 51.84 & 0.65 & 1.98 & 0.89 \\
\hline $40-60$ & 42.52 & 0.29 & 1.24 & 0.32 & $40-60$ & 40.34 & 0.40 & 1.87 & 0.68 \\
\hline $60-80$ & 45.64 & 0.34 & 0.57 & 0.27 & $60-80$ & 40.21 & 0.32 & 1.63 & 0.48 \\
\hline
\end{tabular}


Table.3 Effect of soil characteristics on predictability of micronutrient cations

\begin{tabular}{|c|c|c|}
\hline Depth & Available Fe & $\mathbf{R}^{2} \mathbf{X} 100$ \\
\hline $0-20 \mathrm{~cm}$ & $-93.814+21.667 \mathrm{pH}+2.583 \mathrm{OC}+14.587 \mathrm{Cu}+0.744 \mathrm{Mn}$ & $54.5^{*}$ \\
\hline $20-40 \mathrm{~cm}$ & $-29.601+8.968 * \mathrm{OC}+28.598 * \mathrm{Cu}$ & $55.5^{*}$ \\
\hline $40-60 \mathrm{~cm}$ & $4.375+11.638 * \mathrm{Mg}+48.849 * \mathrm{Cu}$ & $55.9 *$ \\
\hline $60-80 \mathrm{~cm}$ & $20.040+21.483 \mathrm{Cu}+27.590 \mathrm{Zn}$ & $32.2^{*}$ \\
\hline \multicolumn{3}{|c|}{ Available $\mathbf{C u}$} \\
\hline $20-40 \mathrm{~cm}$ & $0.226+0.008 * \mathrm{Fe}$ & $28.8^{*}$ \\
\hline $40-60 \mathrm{~cm}$ & $-0.068+0.005^{*} \mathrm{Fe}+0.663^{*} \mathrm{Zn}$ & $54.5^{*}$ \\
\hline $60-80 \mathrm{~cm}$ & $-0.186+0.005 \mathrm{Fe}+0.986^{*} \mathrm{Zn}$ & $46.3^{*}$ \\
\hline \multicolumn{3}{|c|}{ Available Mn } \\
\hline $0-20 \mathrm{~cm}$ & $-43.436+10.239 \mathrm{pH}+1.595 \mathrm{Ca}+0.042 \mathrm{Fe}$ & $42.4 *$ \\
\hline $20-40 \mathrm{~cm}$ & $-127.146+22.757 * \mathrm{pH}+2.040 * \mathrm{OC}-2.096 \mathrm{Ca}+8.512 \mathrm{Zn}$ & $46.7 *$ \\
\hline $40-60 \mathrm{~cm}$ & $-0.853+2.932 * \mathrm{OC}$ & $30.0^{*}$ \\
\hline $60-80 \mathrm{~cm}$ & $-51.010+10.913^{*} \mathrm{Ph}$ & $20.9 *$ \\
\hline \multicolumn{3}{|c|}{ Available $\mathbf{Z n}$} \\
\hline $0-20 \mathrm{~cm}$ & $0.086+1.145 \mathrm{EC}+0.044 \mathrm{CEC}+0.010$ Clay & $46.2 *$ \\
\hline $40-60 \mathrm{~cm}$ & $0.057+0.002 \mathrm{~K}+0.384 * \mathrm{Cu}$ & $48.4^{*}$ \\
\hline $60-80 \mathrm{~cm}$ & $0.225+0.002 \mathrm{Fe}+0.250 * \mathrm{Cu}$ & $42.1 *$ \\
\hline
\end{tabular}

The multiple correlation and regression analyses indicated that $28.8,54.5$ and 46.3 per cent variability in the respective $2^{\text {nd }}, 3^{\text {rd }}$ and $4^{\text {th }}$ layers may be the combined effect of $\mathrm{Fe}$ and $\mathrm{Zn}$ content in the profiles.

\section{Manganese (Mn)}

DTPA-extractable $\mathrm{Mn}$ in the soil profiles varied from 0.44 to $38.67 \mathrm{mg} \mathrm{kg}^{-1}$ soil. Taking into consideration the critical value of $1.0 \mathrm{mg} \mathrm{Mn}$ $\mathrm{kg}^{-1}$ soil (Lindsay and Norvell, 1978), the studied soil profiles were above the critical limit. The high amount of $\mathrm{Mn}$ in the soils might be due to the solubility of this cation is higher at low $\mathrm{pH}$.

The abundance of DTPA - Mn in soils of Manipur was also reported by Sen et al., (1997), Sarkar et al., (2002), Athokpam et al., (2013), Athokpam et al., (2016) and Athokpam et al., (2018). The surface soils contained higher Mn and decreased with increase in depth. Only 3.75 per cent of the studied samples were below critical limits. It observed significant multiple regression coefficient with $\mathrm{pH}\left(22.757^{*}\right)$ and OC $\left(2.040^{*}\right)$ in the second layer, OC $(2.932 *)$ in the third layer and $\mathrm{pH}(10.913 *)$ in the fourth layer. Multiple correlation and regression analyses indicated that 42.4, 46.7, 30.0 and 20.9 per cent variability of the available Mn content could be attributed to the simultaneous effect of $\mathrm{pH}, \mathrm{Ca}, \mathrm{Fe}, \mathrm{OC}$ and $\mathrm{Zn}$ in the soil profiles.

\section{Zinc (Zn)}

DTPA-extractable $\mathrm{Zn}$ in the soil profiles varied from 0.10 to $1.67 \mathrm{mg} \mathrm{kg}^{-1}$ soil. Sen et al., (1997) reported the available $\mathrm{Zn}$ content varies from 0.2 to $1.4 \mathrm{mg} \mathrm{kg}^{-1}$ and decreased down the profile (Khanday et al., 2017). Similar report was also reported by Athokpam et al., (2016) in the citrus orchard of Ukhrul district, Manipur. Considering $0.6 \mathrm{mg} \mathrm{kg}^{-1}$ as the critical limit of available $\mathrm{Zn}$ as suggested by (Lindsay and Norvell, 1978), 48.75 per cent were sufficient and 51.25 per cent soils were deficient in $\mathrm{Zn}$ status. The low content of this cation might be the lost from the surface soils as the soils are very acidic in most of the profiles. DTPA- 
extractable $\mathrm{Zn}$ showed significant regression with $\mathrm{Cu}\left(0.384^{*}\right)$ in the third layer and also $\mathrm{Cu}$ $(0.250 *)$ in the fourth layer. The multiple regression equations presented in the Table 3 indicate a predictability value of $46.2,48.4$ and 42.1 per cent by all factors taken together in the $1^{\text {st }}, 3^{\text {rd }}$ and $4^{\text {th }}$ layers, respectively.

There exists variation in soil properties with respect to depth in soil profiles. Surface layers tend to have more content of nutrients which gradually decreases downward in most of the profiles especially from $40 \mathrm{~cm}$ to $80 \mathrm{~cm}$ depth. The surface layer soils were high in organic carbon.

The macronutrients content varies from low to medium. For micronutrients, $\mathrm{Fe}, \mathrm{Cu}$, and $\mathrm{Mn}$ were sufficient in most samples. However, some soils were deficient in $\mathrm{Zn}$. This may be one of the important reasons for decreasing production potential of jhum practice in Chandel district of Manipur. Therefore, it is advisable to supply both macro as well as $\mathrm{Zn}$ to the crop to sustain the fertility of the soils as well as productivity of crop.

\section{References}

Athokpam, H., Wani, S.H., Kamei, D., Athokpam H.S., Nongthmaithem, J., Kumar, D., Singh, Y.K., Naorem, B.S., Devi, T.R. and Devi, L. 2013. Soil macro and micro-nutrient status of Senapati district, Manipur (India). African Journal of Agricultural Research. 8(39):49324936.

Athokpam, H.S., Singh, K.S., Chongtham, N, Devi, K.N., Singh, N.B., Sanatomba A. and Sharma, P.T. (2018). Vertical distribution of micronutrient cations in Imphal East and West district, Manipur (India). International Journal of Current Microbiology and Applied Sciences. 7(8): 4222-4227.

Athokpam, H.S., Zimik, V.S, Chongtham, N., Devi, K.N., Singh, N.B., Watham, L., Sharma, P.T. and Athokpam, H. (2016)
Profile distribution micronutrient cations in citrus orchard of Ukhrul district, Manipur (India). International Journal of Agriculture, Environment and Biotechnology. 9(4): 691-697.

Bell, R.W. and Dell, B. (2008). Micronutrients for sustainable food, feed, fibre and bioenergy production. $1^{\text {st }}$ edn. IFA, Paris, France.

Borah, D.K., Bordoloi, P.K., Karmakar, R.M., Baruah, N.G. and Das, M. 1987. Practical Manual of Fundamental of soil Science (Part-III), Jorhat, Assam

Bouyoucos, G.J. 1951. Soil Science. 23: 319343.

Bray, R.H. and Kurtz, L.T. 1945. Determination of total, organic and available forms of phosphorus in soils. Soil Science. 59: 3945.

Gao, S., Yan, R., Cao, M., Yang, W., Wang, S. and Chen, F. 2008. Effect of on growth, antioxidant enzymes and phenylalanine ammonia-lyase activities in Jatropha curcas L. seedling. Plant, Soil and Environment. 54(3): 117-122.

Gupta, N., Trivedi, S.K., Bansali, K.N., Kaul, R.K. 2003. Vertical distribution of micronutrient cations in some soil series of north Madhya Pradesh. Journal of the Indian Society of Soil Science. 51: 517522.

Gupta, P.K. 2006. Soil pH and Electrical Conductivity. Soil Plant Water and Fertilizer Analysis, pp. 81-89.

Jackson, M.L. 1973. Soil Chemical Analysis. Prentice-Hall of India Pvt. Ltd. New Delhi.

Khanday, M.U.D.D., Ram, D., Wani, J.A. and Ali, T. 2017. Vertical distribution of nutrients of the soils of Namblan subcatchment of Jhelum basin of Srinagar district in Kashmir valley. International Journal of Current Microbiology and Applied Sciences. 6: 375-381.

Kumar, P., Sharma, S.P. and Sharma, P.O. (1996). Micronutrient status of different landforms of Soan River valley soils of lower Shiwaliks. Journal of the Indian Society of Soil Science. 44(2): 330-331. 
Lindsay, W.L. and Norvell, W.A. (1978). Development of a DTPA soil test for zinc, iron, manganese and copper. Soil Science Society of American Journal. 42: 421428.

Murthy, I.Y.L.N., Sastry, T.G., Dutta, S.C., Naranasawy, G. and Rattan, R.K. 1997. Distribution of micronutrient cations in Vertisols derived from different parent materials. Journal of the Indian Society of Soil Science. 45(3): 577-580.

Panse, V.G. and Sukhatme, P.V. (1961). Statistical methods for agricultural workers, ICAR, New Delhi.

Pati, R. and Mukhopadhyay, D. (2011). Distribution of cationic micronutrients in some acid soils of West Bengal. Journal of the Indian Society of Soil Science. 59(2): 125-133.

Rengel, Z. (2007). Cycling of micronutrients in terrestrial ecosystems. In: Marschner, P. and Rengel, Z. (Eds.) Nutrient Cycling in Terrestrial Ecosystem. Springer- Verlag, Berlin, Heidelberg, pp. 93-121.

Sangwan, B.S. and Singh, K. (1993). Vertical distribution of $\mathrm{Zn}, \mathrm{Mn}, \mathrm{Cu}$ and $\mathrm{Fe}$ in the semi- arid soils of Haryana and their relationships with soil properties. Journal of the Indian Society of Soil Science. 41(3): 463-467.

Sarkar, D., Baruah, U., Gangopadhyay, S.K., Sahoo, A. K., Velayutham, M. (2002). Characteristics and classification of soils of Loktak catchment area of Manipur for sustainable land use planning. Journal of the Indian Society of Soil Science. 50(2): 196-204.

Satyavathi, P.L.A. and Reddy, M.S. (2004). Distribution of DTPA extractable micronutrient in soils of Telangana, Andhra Pradesh. Agropedology. 14(1): 32-37.
Sen, T.K., Dubey, P.N., Maji, A.K. and Chamuah, G.S. (1997). Status of micronutrients in some dominant soils of Manipur. Journal of the Indian Society of Soil Science. 45(2): 388-390.

Sharma, R.P., Singh, M. and Sharma, J.P. (2003). Correlation studies on micronutrients vis-à-vis soil properties in some soils of Nagaur district in semi-arid region of Rajasthan. Journal of the Indian Society of Soil Science. 51(4): 522-527.

Singh, K.M.S. and Dhankar, S.S. (1989). Influence of soil characteristics on profile distribution of DTPA- extractable micronutrient cations. Indian Journal of Agricultural Sciences. 59: 331-334.

Singh, M.V. (2004). Micronutrient deficiencies in Indian soils and field usable practices for their correction. IFA International Conference on Micronutrients, New Delhi.

Subbiah, B.V. and Asija, G.L. 1956. A rapid procedure for estimation of available $\mathrm{N}$ in soils. Current Science. 25: 259-260.

Verma, V.K., Setia, R.K., Sharma, P.K. (2007b). Distribution of micronutrient cations in different physiographic units of semi-arid region of Punjab. Agropedology. 18: 58-65.

Verma, V.K., Setia, R.K., Sharma, P.K. Khurana, M.P.S. and Kang, G.S. (2007a). Pedospheric distribution of micronutrient cations in soil developed on various landforms in north-east Punjab. Journal of the Indian Society of Soil Science. 55: 515-520.

Walkley, A. and Black, C.A. (1934). An examination of the method for determining soil organic matter and a proposed modification of the chromic acid titration method. Soil Science. 37(16): 29-39.

\section{How to cite this article:}

Jurist Anal Hrangbung, Herojit Singh Athokpam, S. Jekendra Singh, K. Nandini Devi and Gopimohan Singh, N. 2018. Profile Distribution of Micronutrient Cations in Soils under Jhum Land in Chandel District of Manipur (India). Int.J.Curr.Microbiol.App.Sci. 7(11): 332-340. doi: https://doi.org/10.20546/ijcmas.2018.711.042 